Acta Crystallographica Section D

Biological

Crystallography

ISSN 0907-4449

Núria Valls, ${ }^{a}$ Glenford Wright, ${ }^{a}$ Roberto A. Steiner, ${ }^{b}$ Garib N. Murshudov ${ }^{b}$ and Juan A. Subirana ${ }^{\mathrm{a} *}$

àepartament d'Enginyeria Química, Universitat Politècnica de Catalunya, Avinguda Diagonal 647, E-08028 Barcelona, Spain, and ${ }^{\mathbf{b}}$ York Structural Biology Laboratory, Department of Chemistry, University of York, York Y010 5YW, England

Correspondence e-mail:

juan.a.subirana@upc.es
C) 2004 International Union of Crystallography

Printed in Denmark - all rights reserved

\title{
DNA variability in five crystal structures of d(CGCAATTGCG)
}

The deoxyoligonucleotide d(CGCAATTGCG) has previously been crystallized in four different space groups. The crystals diffract to moderate resolution (2.3-2.9 $\AA$ ). Here, a fifth crystal form that diffracts to higher resolution $(1.6 \AA)$ is presented which was obtained thanks to the use of $\mathrm{Co}^{2+}$ and cryogenic temperatures. The availability of five different crystal structures allows a thorough analysis of the conformational variability of this DNA sequence. It is concluded that the central hexamer sequence CAATTG has a practically constant conformation under all conditions, whilst the terminal base pairs at both ends vary considerably as a result of differing interactions in the crystals. The new crystal structure presented here is stabilized by guanine- $\mathrm{Co}^{2+}-$ guanine interactions and the formation of $\mathrm{C1}^{+}-\mathrm{G} 8 \cdot \mathrm{C} 3$ triplexes between neighbouring duplexes. As a result of the higher resolution of the crystal structure, a more regular structure was obtained and a clear definition of the spine of hydration was observed which was not visible in the four previous structures.

\section{Introduction}

The d(CGCAATTGCG) $)_{2}$ duplex has been subjected to several crystallization attempts with different minor-groovebinding drugs (Spink et al., 1995; Nunn et al., 1997; Wood et al., 1997) and peptides (Soler-López et al., 2002). Except in the case of the drug netropsin (Nunn et al., 1997), complexes have not yet been obtained. Crystallization was carried out in the presence of either $\mathrm{Mg}^{2+}$ or $\mathrm{Zn}^{2+}$ ions. Each crystallization experiment yielded a different space group. This indicates the effect of variable packing forces as a function of the presence of either ions, drugs or peptides. In all cases MPD was used as a precipitant.

Prompted by our finding that $N$ - $\alpha$-(9-acridinoyl)-tetraarginine intercalates in an AA/TT step of the dodecamer duplex d(CGCGAATTCGCG) (Malinina et al., 2002), we have tried to obtain a complex of d(CGCAATTGCG) with an acridine compound. However, no intercalation was found in the crystals obtained. We carried out several crystallization attempts. The best diffracting crystals $(1.6 \AA)$ were obtained using $\mathrm{Co}^{2+}$ ions. This metal ion participates in guanine- $\mathrm{Co}^{2+}$ guanine bridges in the crystal.

In this paper, we describe the structure of the $\mathrm{d}(\text { CGCAATTGCG })_{2}$ duplex at $1.6 \AA$. This is the most accurate structure of this decamer achieved to date. In addition, we compare this structure with the other four available structures. This is a unique case in which the same oligonucleotide has been crystallized in five different environments. We will try to
Received 3 November 2003 Accepted 5 February 2004

PDB Reference: d(CGCAATTGCG), 1s23, r1s23sf.

NDB Reference: BD0066. 
ascertain the type of interactions that stabilize each crystal structure. We can also determine the variability of the oligonucleotide when found in different environments.

\section{Materials and methods}

\subsection{Crystallization}

The deoxydecanucleotide d(CGCAATTGCG) was supplied by Oswell DNA service (Southampton, UK) as the ammonium salt. The intercalating drug acridine-(Arg-GlyArg) was synthesized by solid-phase methods. After the Bocpeptide was assembled on a $p$-methylbenzhydrylamine resin, the N-terminus was deprotected and 9-acridinecarboxylic acid was coupled by means of PyBOP and diisopropylethylamine (5, 5 and 10 equivalents, respectively) in 1:1 DMSO/DMF for $1 \mathrm{~h}$. Acidolysis with HF/anisole $(9: 1,273 \mathrm{~K}, 1 \mathrm{~h})$ provided the target compound in a highly homogeneous form ( $>95 \%$ by HPLC) and with a correct MALDI-TOF mass spectrum.

Crystals were grown by vapour diffusion in a hanging drop containing $0.5 \mathrm{~m} M$ DNA duplex, $1 \mathrm{~m} M$ acridine-(Arg-GlyArg) drug adduct, $20 \mathrm{~m} M$ sodium cacodylate buffer $\mathrm{pH} 6.5$, $4 \mathrm{~m} M \mathrm{CaCl}_{2}, 1 \mathrm{~m} M \mathrm{CoCl}_{2}, 0.5 \mathrm{~m} M$ spermine and 10\% MPD equilibrated against a $25 \%$ MPD reservoir. Colourless crystals grew at $293 \mathrm{~K}$ in approximately three weeks to typical dimensions of $0.2 \times 0.06 \times 0.06 \mathrm{~mm}$.

\subsection{X-ray data collection}

Crystals were flash-cooled at $120 \mathrm{~K}$ and diffraction data were collected on a MAR CCD detector using synchrotron radiation at ESRF beamline BM14. Two data sets were collected at resolution cutoffs of 2.25 and $1.6 \AA$, respectively. The low-resolution pass was needed to accurately measure the high-intensity reflections, which resulted in detector saturation in the high-resolution pass. The data were integrated and scaled with the $H K L$ suite (Otwinowski \& Minor, 1997).

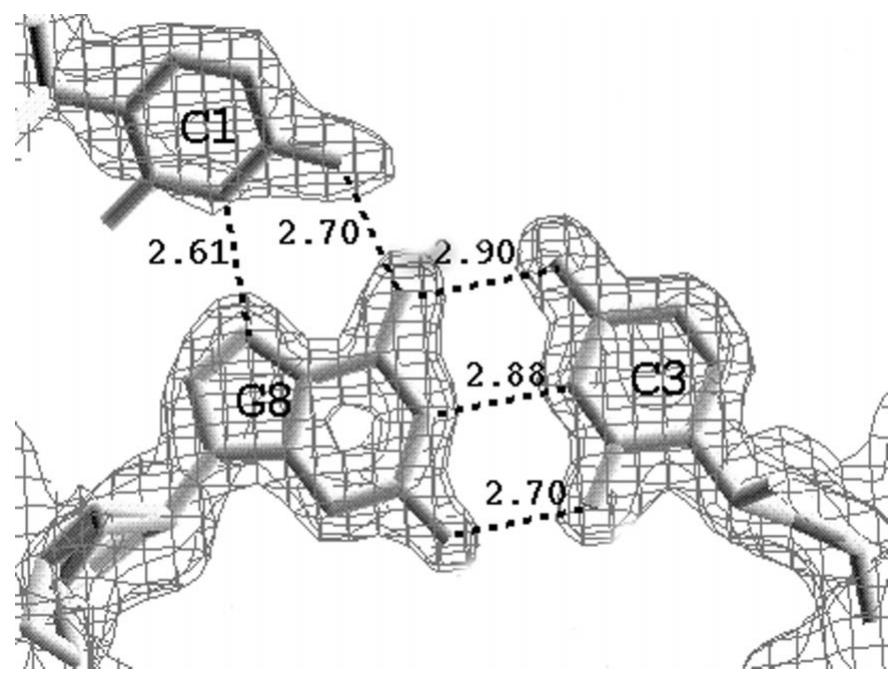

Figure 1

Electron-density map at the $1 \sigma$ level of the flipped-out base $\mathrm{C} 1$ which forms a triplet with base pair C3.G8 in a neighbouring duplex. Hydrogen bonds are shown as dashed lines with distances in $\AA$. Note that the triplet is not planar, as is apparent from Fig. 3 .
Table 1

Crystallographic and refinement statistics.

Values in parentheses are for the last resolution shell.

\begin{tabular}{|c|c|}
\hline Space group & $I 2_{1} 2_{1} 2_{1}$ \\
\hline Unit-cell parameters & $a=26.22, b=44.36, c=52.34$ \\
\hline Asymmetric unit contents & One DNA strand, $0.5 \mathrm{Co}^{2+}$ ions, $46 \mathrm{H}_{2} \mathrm{O}$ \\
\hline Temperature (K) & 120 \\
\hline Resolution range $(\AA)$ & $25-1.6(1.66-1.6)$ \\
\hline Completeness (\%) & $98.4(89.1)$ \\
\hline Unique reflections & 4172 \\
\hline Overall redundancy $\dagger$ & 17.7 \\
\hline$R_{\text {sym }} \neq$ & $0.077(0.60)$ \\
\hline$R_{\text {work }} \S / R_{\text {free }}$ & $0.21 / 0.281$ \\
\hline$R_{\text {work }}$ (all reflections) & 0.214 \\
\hline
\end{tabular}

$\dagger$ Total reflections registered divided by the number of unique reflections. $\ddagger R_{\text {sym }}(I)=$ $\sum_{h k l} \sum_{j}\left|I_{j}(h k l)-\langle I(h k l)\rangle\right| / \sum_{h k l} \sum_{j} I_{j}(h k l) \quad$ calculated for the whole data set. $\$ \sum_{h k l}\left|F_{\mathrm{o}}(h k l)-k F_{\mathrm{c}}(h k l)\right| / \sum_{h k l} F_{\mathrm{o}}(h k l)$. $\quad R$ factor of reflections used for cross-validation in the refinement.

Crystallographic data and refinement statistics are given in Table 1.

\subsection{Structure determination and refinement}

The structure of d(CGCAATTGCG) was determined by the molecular-replacement method using the program EPMR v.2.5 (Kissinger et al., 1999) starting from the low-resolution model (NDB code UDJ031) published by Spink et al. (1995). Data in the resolution range $15-3 \AA$ were used to determine the molecular-replacement solution. Bases in the decamer were numbered 1-10 starting with the cytosine at the $5^{\prime}$ end. The terminal bases $\mathrm{C} 1$ and G10 were not used in the model since they were not part of the duplex. The initial model was subjected to rigid-body refinement using the program CNS v.1.1 (Brünger et al., 1998) with data in the resolution range 25-2.0 А. At this stage, $10 \%$ of the unique reflections were set apart to calculate a free $R$ factor (Brünger, 1992) as an independent cross-validation indicator of the progress of refinement. After several cycles of refinement, electron density clearly showed the base G10 and an ion that was modelled as $\mathrm{Co}^{2+}$. Extra density for the $\mathrm{C} 1$ base was seen next to $\mathrm{G} 2$. However, it was not possible to model its exact position at this stage. Refinement was continued using the program REFMAC5 (Murshudov et al., 1997) from the CCP4 suite (Collaborative Computational Project, Number 4, 1994) using the same resolution limits. After several cycles of maximumlikelihood isotropic restrained refinement, the $\mathrm{C} 1$ base appeared clearly with a flipped-out geometry in $m F_{\mathrm{o}}-D F_{\mathrm{c}}$ and $2 m F_{\mathrm{o}}-D F_{\mathrm{c}}$ electron-density maps. At this stage of refinement, water molecules were added to the model at positions corresponding to peaks in the difference Fourier higher than the $3 \sigma$ level if they satisfied hydrogen-bonding criteria. Additional water molecules could be located in the electron-density maps by including the high-resolution data (to $1.6 \AA$ ). This also allowed a more reliable inclusion in the refinement of $\mathrm{H}$ atoms at their riding positions. We also found two positions for the phosphate of C9 and the sugar of G8. Note that data in the last shells contained reflections with a significant intensity $(>3 \sigma)$ which contributed to the improvement of the electron-density map. Manual rebuilding was 
carried out with the programs TURBO-FRODO (Roussel et al., 1998) and QUANTA (Accelrys Inc.). The refinement converged at $R=21.0 \%$ and $R_{\text {free }}=28.1 \%$ for the resolution range 25-1.6 A. Further refinement details are given in Table 1 . No residual electron density was found that could be attributed to the drug. An example of the quality of the final electron-density map in the $\mathrm{C} 1$ region is shown in Fig. 1. This figure was prepared with the program SETOR (Evans, 1993). Figs. 2-5 were drawn with the program Cerius2 (Accelrys Inc.).

\section{Results}

\subsection{Description of the present structure}

The oligonucleotide d(CGCAATTGCG) has been crystallized in space group $I 2_{1} 22_{1}$ with one strand in the asymmetric unit. Continuous columns of duplexes in the B-DNA conformation with antiparallel Watson-Crick base pairs are formed for eight bases of the decamer (the packing of the crystal is shown in Fig. 2). The terminal bases C1 and G10 are flipped out. Columns of duplexes are linked through a $\mathrm{Co}^{2+}$ ion that interacts with two flipped-out guanines from neighbouring molecules with the N7 of G10 at a typical distance of $2.2 \AA$ from the metal ion. The interaction of the $\mathrm{Co}^{2+}$ ion has previously been described in other oligonucleotide structures

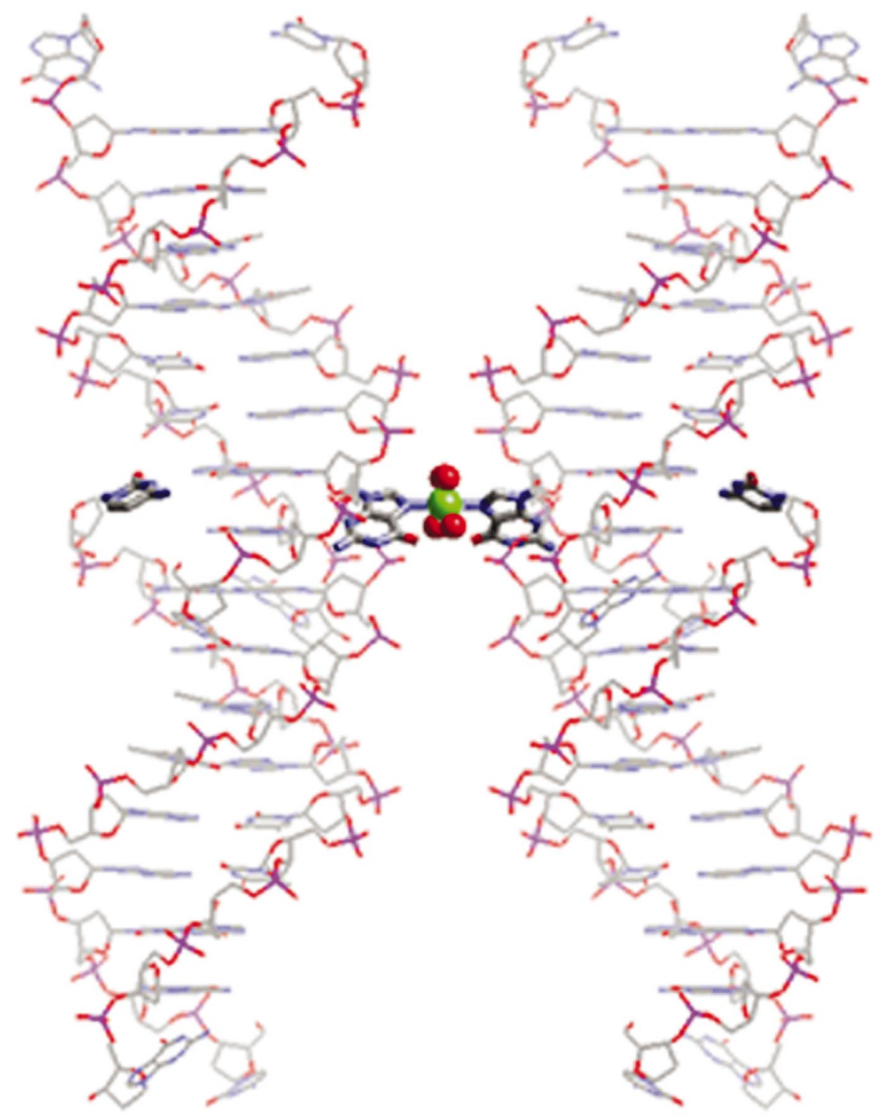

Figure 2

Two neighbouring columns in the crystal structure. A cobalt ion (green) interacts with the N7 atom of two guanines. The bonds of the flipped-out residues in the central region are shown with a larger diameter. Hydration waters associated with the $\mathrm{Co}^{2+}$ ion are represented as red spheres. in the presence of either $\mathrm{Co}^{2+}$ (Yang et al., 2000) or $\mathrm{Ni}^{2+}$ (Abrescia et al., 1999). Thus, the present structure adds a new crystallization environment for this oligonucleotide. Our work also shows that $\mathrm{Co}^{2+}$ ions may be useful to obtain crystals that diffract to high resolution, since they form intermolecular bridges between symmetry-related molecules.

The terminal base G10 enters the minor groove of the next duplex in a column. It is hydrogen bonded to G2. Since its description by Spink et al. (1995), this type of interaction has been frequently found in different structures as reviewed by Subirana \& Abrescia (2000).

The terminal cytosine (C1) is also flipped out. This observation is clearly supported by electron-density maps (Fig. 1). However, the average $B$ factor for this base suggests a greater disorder compared with the rest of the structure. $\mathrm{C} 1$ is protonated at $\mathrm{N} 3$ and interacts with G8 by hydrogen bonding. The interactions of the terminal bases are shown in detail in Fig. 3.

The oligonucleotide crystal contains an extensive network of water molecules, some of which form bridges between neighbouring oligonucleotide columns. All phosphate groups are hydrogen bonded to water molecules. Most bases also show direct hydrogen bonds to water molecules. An extensive network covers the major-groove side of the central AATT region. A well defined water spine is found at the centre of the minor groove, as shown in Fig. 4. It is virtually identical to that described in other high-resolution structures of oligonucleotides that also contain a central AATT sequence (Soler-López et al., 1999).

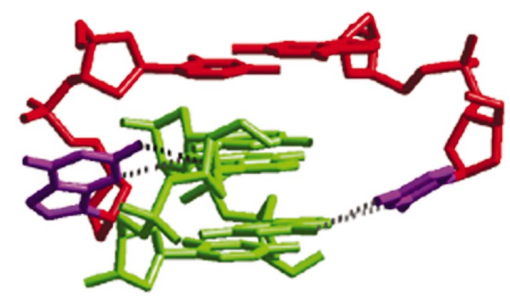

Figure 3

A detailed view of the interaction between the ends of two neighbouring duplexes (red and green) in a column. The flipped-out residues ( $\mathrm{C} 1$ and G10) are shown in purple. Hydrogen bonds between G10 and G2 and between $\mathrm{C} 1$ and G8 are indicated as dashed lines.

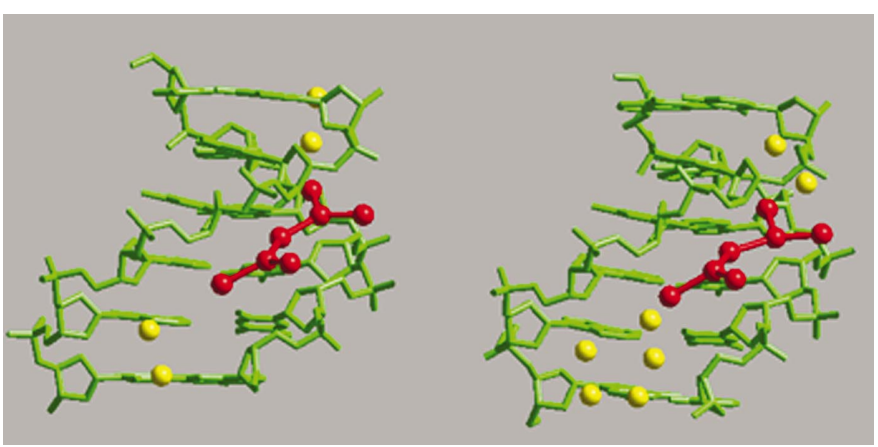

Figure 4

Comparison of the hydration spine in our structure (left) with the spine present in d(GCGAATTCG) (right) (Soler-López et al., 1999). Red spheres indicate waters in practically identical positions, with hydrogen bonds between them shown as virtual bonds. Hydration waters away from the central region (yellow) have different positions. 
Table 2

Structural features of d(CGCAATTGCG) in different crystal forms.

\begin{tabular}{|c|c|c|c|c|c|c|c|c|c|c|c|}
\hline Structure & $\begin{array}{l}\text { NDB } \\
\text { code }\end{array}$ & $\begin{array}{l}\text { Space } \\
\text { group }\end{array}$ & $\begin{array}{l}\text { Contents } \\
\text { of } \mathrm{AU}\end{array}$ & $\begin{array}{l}\text { Resolution } \\
(\AA)\end{array}$ & Ions & $\mathrm{C} 1$ & G10 & $\left\langle D_{z}\right\rangle \dagger(\AA)$ & $\begin{array}{l}\text { Minor-groove } \\
\text { width } \ddagger(\AA)\end{array}$ & $\begin{array}{l}\text { Volume } \\
\text { per bp } \\
\left(\AA^{3}\right)\end{array}$ & Reference \\
\hline 1 & UDJ031 & $I 2_{1} 2_{1} 2_{1}$ & One strand & 2.5 & $\mathrm{Mg}^{2+}$ & Flipped out & Minor groove & $3.27(0.07)$ & 3.8 & 1432 & Spink et al. (1995) \\
\hline 3 & BDJ069 & $C 2$ & Duplex & 2.3 & $\mathrm{Mg}^{+2}$ & Duplex & Duplex & $3.37(0.35)$ & 5.0 & 1237 & Wood et al. (1997) \\
\hline 4 & UD0012 & $C 222_{1}$ & One strand & 2.9 & $\begin{array}{l}\mathrm{Zn}^{+2}, \\
\quad \text { peptide }\end{array}$ & Flipped out & Minor groove & $3.34(0.10)$ & 3.1 & 1638 & Soler-López et al. (2002) \\
\hline
\end{tabular}

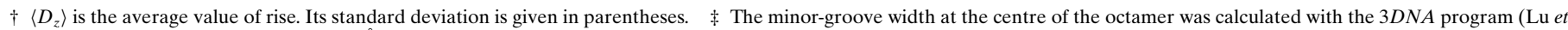
al., 2003). The van der Waals radius $(5.8 \AA)$ of the phosphate group was substracted.

\subsection{Comparison with other structures}

3.2.1. General features. Various studies of the oligonucleotide that we have crystallized are summarized in Table 2. The central octamers of the five structures are compared in Fig. 5. All of them are rather similar, particularly in the central region. The main differences are found in the phosphodiester backbone of both ends, as shown in Fig. 5. It should be noted that only structure 3 has all bases in a duplex conformation; all other structures have the terminal $\mathrm{C} 1$ and G10 bases flipped out and interacting with neighbouring duplexes.

Structures 1-3 in Table 2 were collected at room temperature and are expected to have higher disorder than structures

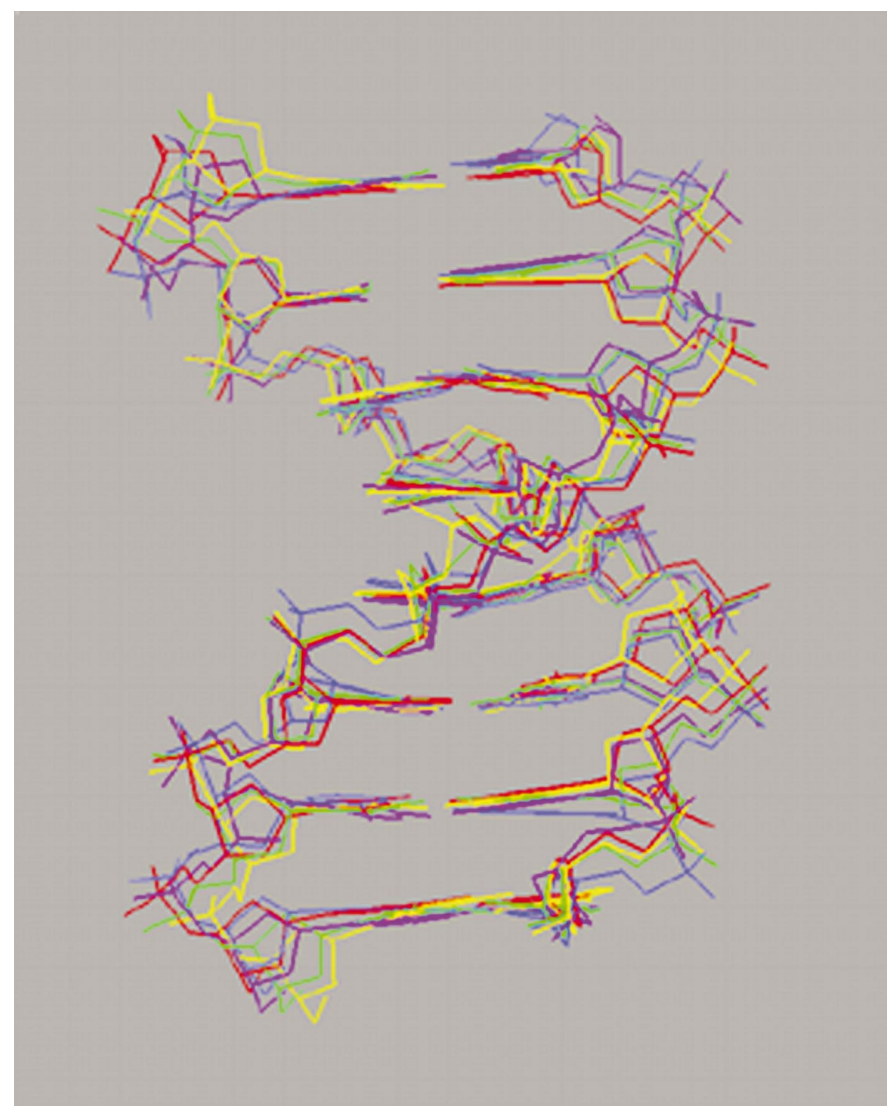

Figure 5

Superposition of all structures. Structure 1 (green) has been taken as reference. Colours correspond to the following structures: 2, blue; 3 , purple; 4, yellow; 5 , red.
4 and 5, which were collected at $120 \mathrm{~K}$. However, structure 4 also shows high disorder owing to the presence of a mobile peptide moiety which contains four arginines. The presence of the peptide also gives a higher volume per base pair, as shown in Table 2.

3.2.2. Packing. All structures reported in Table 2 display similar packing, except for structure 3 , which crystallized with a very different symmetry in space group $C 2$. The duplexes also packed in layers of parallel columns, but the helices in neighbouring layers have their axes crossed at an angle of $42^{\circ}$ (Wood et al., 1997). Structures 1 and 5 were crystallized under very different conditions. However, the structures are almost identical. They both have the base G10 flipped out. In structure 5 the base $\mathrm{G} 10$ interacts with a $\mathrm{Co}^{2+}$ ion, whereas in structure 1 water molecules connect the different columns in a way similar to that shown in Fig. 2. Base $\mathrm{C} 1$ also presents a different position. In our structure the interaction $\mathrm{C} 1-\mathrm{G} 8$ through two hydrogen bonds is clearly seen, while in structure 1 the base $\mathrm{C} 1$ was very disordered and tentatively modelled as interacting with the base pair G2.C9 of the next duplex. The higher resolution of structure 5 may help to define a more accurate position for the base $\mathrm{C} 1$. The netropsin structure 2 has $\mathrm{C} 1$ in the same position as shown in Fig. 1, whereas in structure 4 it shows high disorder owing to the fact that it is bound to the disordered peptide chain.

In all cases except structure 3, the terminal guanines G10 enter the minor groove of the next duplex in the oligonucleotide columns as shown in Fig. 2 and in more detail in Fig. 3.

The oligonucleotide columns in structures 1 and 5 have the same relative positions, as shown in Fig. 2, whereas in structures 2 and 4 they are also parallel but displaced; there is no guanine-guanine interaction between neighbouring columns.

Ions play a very important role in crystallization. Crystals 1 , 2 and 3 contain $\mathrm{Mg}^{2+}$ but no ion was present in the final refined structures. $\mathrm{Zn}^{2+}$ and $\mathrm{Co}^{2+}$ are clearly seen in structures 4 and 5, respectively. In crystal 4 , two kinds of $\mathrm{Zn}^{2+}$ ions were found: one interacts with the oxygen of phosphates and the other interacts with $\mathrm{N} 7$ of a single guanine; it does not form a bridge between columns.

3.2.3. Minor-groove structure. In general (Table 2), all five decamers show the narrow minor groove typically found in AT-rich sequences, but because of the presence of netropsin, 
Table 3

Twist $\left(^{\circ}\right)$ of individual base steps in d(CGCAATTGCG).

Only the central octamer in the duplex conformation is analyzed. Expected values and their standard deviation are taken from Subirana \& Faria (1997). Values which deviate by more than two standard deviations are shown in bold. Note that in structures 2 and 3 the asymmetric unit is the whole duplex instead of a single strand. Therefore, there are two values for each step, except in the central ApT.

\begin{tabular}{llllll}
\hline Structure & $\mathrm{GpC}$ & $\mathrm{CpA} / \mathrm{TpG}$ & $\mathrm{ApA} / \mathrm{TpT}$ & $\mathrm{ApT}$ & $\langle\Omega\rangle \dagger$ \\
\hline 1 & 37.4 & 38.1 & 30.0 & $\mathbf{4 1 . 5}$ & $36.1(4.4)$ \\
2 & $34.0 / 38.2$ & $32.3 / 32.3$ & $37.1 / 37.2$ & 34.5 & $35.1(2.4)$ \\
3 & $\mathbf{2 4 . 9} / 37.9$ & $35.8 / 38.7$ & $38.2 / 39.6$ & 30.4 & $35.1(5.4)$ \\
4 & 39.0 & 31.7 & $\mathbf{4 3 . 3}$ & 29.6 & $36.8(5.7)$ \\
5 & $\mathbf{3 1 . 8}$ & 33.2 & 36.2 & 34.9 & $33.9(1.8)$ \\
Expected & $37.5(2.7)$ & $38.5(8.6)$ & $35.3(3.2)$ & $32.0(2.8)$ & \\
\hline
\end{tabular}

$\dagger$ Average twist value, with standard deviation in parentheses.

structure 2 has a wider minor groove. In the case of structure 3, the interaction with neighbouring duplexes by interpenetration of their grooves also results in a wider minor groove.

3.2.4. Structural details. As shown in Fig. 5, all five structures are rather similar; the r.m.s. deviations between the central octamers are rather small. They range between $0.71 \AA$ (for structures 1 and 3) and $1.22 \AA$ (for structures 2 and 4). The conformational parameters of all structures were compared using the data available in the NDB (Berman et al., 1992). Those for the present structure were calculated with $3 D N A$ v.1.5 (Lu et al., 2003). Only small differences were found between the structures, with the exception of the structure 3, in which several phosphates adopted the BII conformation. The central A.T base pairs all show a large negative propeller twist (around $-14^{\circ}$ ) as expected for this sequence. As a result, the distance between the $\mathrm{C} 2$ atoms of adenine and the $\mathrm{O} 2$ atom of thymine in neighbouring base pairs (AA/TT steps) of our structure is shortened to $3.27 \AA$, which may be considered to be an additional weak hydrogen bond (Madhumalar \& Bansal, 2003). However, the angle $\mathrm{C} 2-\mathrm{H} 2 \cdots \mathrm{O} 2$ is only $114^{\circ}$, which is not optimal for a hydrogen bond.
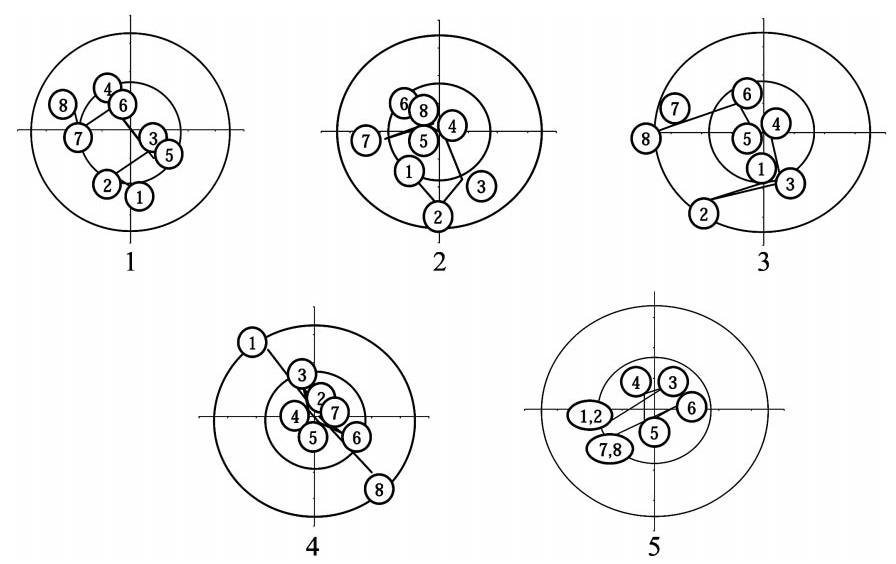

Figure 6

Plots of base-pair normal vectors for the central octamer in all five structures. Inner and outer circles represent angles of 5 and $10^{\circ}$ with respect to the centre of the duplex (between base pairs 4 and 5). Deviations are larger at either end of the duplexes.
As an example of the small differences found, the twist values are compared in Table 3 for all five structures. Most of them fall within the expected average range, with some exceptions that are indicated in bold in the table. These differences probably arise from the variable interactions of the oligonucleotides in the crystal, although some of them, in particular the unexpected changes in the central AATT region, might arise from inaccuracy of the coordinates related to the low resolutions of some of the structures. It is also worth noting that the twist values for the CA/TG step are rather constant. This is surprising as this is a very variable step (Subirana \& Faria, 1997).

An interesting feature of our structure 5 is that the variations in twist (Table 3) and in the conformational angles of all bases are rather small (results not shown), which we attribute to the higher accuracy of the coordinates related to higher resolution of our structure. It has a regular duplex structure with small local variations independent of the local sequence.

In Fig. 6 we also present a bending plot of the central octamer. It can be noted that the central region is rather straight; bending is only detected in the terminal base pairs, which should be attributable to their variable interactions in the crystal structure.

\section{Discussion}

One of the main conclusions of the comparison we have presented is that the crystallization conditions do not have a great influence on the overall conformation of the oligonucleotide except at the ends, which show variable interactions in the crystal. On the other hand, it is not clear why the crystallization conditions have such a strong influence on the crystal packing. For example, structures 1 and 3 were crystallized under very similar conditions. A different drug was present, but it was not incorporated in the crystal lattice. In this respect our structure was also surprising, as under similar crystallization conditions using $\mathrm{Ni}^{2+}$ instead of $\mathrm{Co}^{2+}$ a related decamer crystallized in space group $P 4_{1} 2{ }_{1} 2$ (Abrescia et al., 1999). Flipped-out guanines formed similar ion bridges between neighbouring columns, but instead of being parallel as shown in Fig. 2, they were perpendicular when $\mathrm{Ni}^{2+}$ was used.

The high resolution achieved for crystal 5 allows us to very clearly visualize a spine of hydration (Fig. 4). This was not seen in all previous low-resolution structures of the decamer. Interestingly, related structures solved at similar resolution instead display a well defined spine of hydration as in Vlieghe et al. (1996).

It seems plausible that cryogenic temperatures may be required to stabilize the water molecules. In general, it appears that our structure, which has the highest resolution, is less variable than the others.

We are grateful to Drs G. Parkinson and L. Malinina for valuable help in the molecular-replacement stage. We thank Drs L. Campos and C. Cáceres for help and discussion. NV 
acknowledges a fellowship from the Generalitat de Catalunya. This work has been supported by grants BI2002-00317 from the Ministerio de Ciencia y Tecnología and 2001 SGR 00250 from the Generalitat de Catalunya. Support from the European Project HPRN-CT-2000-00009 is also acknowledged. We thank the BM14 Spanish beamline staff of ESRF (Grenoble) for assistance with data collection. RAS and GNM thank the Wellcome Trust for support.

\section{References}

Abrescia, N. G. A., Malinina, L., Fernandez, L. G., Huynh-Dinh, T., Neidle, S. \& Subirana, J. A. (1999). Nucleic Acids Res. 27, 15931599.

Berman, H. M., Olson, W. K., Beveridge, D. L., Westbrook, J., Gelbin, A., Demeny, T., Hsieh, S.-H., Srinivasan, A. R. \& Schneider, B. (1992). Biophys. J. 63, 751-759.

Brünger, A. T. (1992). Nature (London), 355, 472-475.

Brünger, A. T., Adams, P. D., Clore, G. M., DeLano, W. L., Gros, P., Grosse-Kunstleve, R. W., Jiang, J.-S., Kuszewski, J., Nilges, M., Pannu, N. S., Read, R. J., Rice, L. M., Simonson, T. \& Warren, G. L. (1998) Acta Cryst. D54, 905-921.

Collaborative Computational Project, Number 4 (1994). Acta Cryst. D50, 760-763.

Evans, S. V. (1993). J. Mol. Graph. 11, 134-138.

Kissinger, C. R., Gehlhaar, D. K. \& Fogel, B. (1999). Acta Cryst. D55, 484-491.
Lu, X.-J., Shakked, Z. \& Olson, W. K. (2003). Nucleic Acids Res. 31, 5108-5121.

Madhumalar, A. \& Bansal, M. (2003). Biophys. J. 85, 1805-1816.

Malinina, L., Soler-López, M., Aymamí, J. \& Subirana, J. A. (2002). Biochemistry, 41, 9341-9348.

Murshudov, G. N., Vagin, A. A. \& Dodson, E. J. (1997). Acta Cryst. D53, 240-255.

Nunn, C. M., Garman, E. \& Neidle, S. (1997). Biochemistry, 36, 47924799.

Otwinowski, Z. \& Minor, W. (1997). Methods Enzymol. 276, 307-326.

Roussel, A., Inisan, A. G., Knoops-Mouthuy, E. \& Cambillau, E. (1998). TURBO-FRODO version OpenGL.1. University of Marseille.

Soler-López, M., Malinina, L., Liu, J., Huynh-Dinh, T. \& Subirana, J. A. (1999). J. Biol. Chem. 274, 23683-23686.

Soler-López, M., Malinina, L., Tereshko, V., Zarytova, V. \& Subirana, J. A. (2002). J. Biol. Inorg. Chem. 7, 533-538.

Spink, N., Nunn, C. M., Vojtechovsky, J., Berman, M. \& Neidle, S. (1995). Proc. Natl Acad. Sci. USA, 92, 10767-10771.

Subirana, J. A. \& Abrescia, N. G. A. (2000). Biophys. Chem. 86, 179189.

Subirana, J. A. \& Faria, T. (1997). Biophys. J. 73, 333-338.

Vlieghe, D., Van Meervelt, L., Dautant, A., Gallois, B., Précigoux, G. \& Kennard, O. (1996). Acta Cryst. D52, 766-775.

Wood, A. A., Nunn, C. M., Trent, J. O. \& Neidle, S. (1997). J. Mol. Biol. 269, 827-841.

Yang, X.-L., Robinson, H., Gao, Y.-G. \& Wang, A. H.-J. (2000). Biochemistry, 39, 10950-10957. 